Jefferson de Oliveira Gomes gomes@ita.br

\author{
Adelson Ribeiro de Almeida Jr. \\ adelsonj@ita.br
}

\author{
Alex Sandro de Araújo Silva \\ alexs@ita.br \\ Instituto Tecnológico de Aeronáutica - ITA \\ 12228-900 São José dos Campos, SP, Brazil
}

\author{
Guilherme Oliveira de Souza \\ guilhermeos@cimatec.fieb.org.br \\ SENAI CIMATEC \\ 41650-010 Salvador, BA, Brazil \\ Acson Machado Nunes \\ jacson@ita.br \\ Instituto Tecnológico de Aeronáutica - ITA \\ 12228-900 São José dos Campos, SP, Brazil
}

\section{Evaluation of 5-Axis HSC Dynamic Behavior When Milling TiAl6V4 Blades}

Gas combustion represents the second principal way of electricity generation due to its disposal, and smaller dimension and pollution capacity when compared with diesel engines. The conversion of natural gas in electricity is made through gas turbines, and the rotors of its compressors are components that present significant difficulty in its manufacture. This paper focuses on an optimization approach of the 5-axis milling of titanium blisks, based on all the CAD/CAM modeling chain. Special interest is pointed to the systematic analysis of the correlation between machining parameter and surface integrity properties. The experimental part of this work is composed by two tests. The first one analyses the roughing operation of a TiAl6V4 impeller when using a tool radial engagement of $30 \%$ and $68 \%$. The second is the simultaneous 5 axes milling of a testpiece, a TiAl6V4 integral bladed disk (BLISK) section containing five blades, with which the best milling strategy for this purpose is sought. The results showed the fundamental importance of CAD modeling in order to achieve simultaneous 5 axes milling without intermittent feed speed. It was observed that milling a blade following its parametric curves in the BLISK axis direction is the best choice, among the strategies proposed. Keywords: 5-axis milling, Ti alloys, machinability

\section{Introduction}

Milling is a machining process during which material is removed using a rotating cutting tool. Application areas of milling free-formed surfaces are, for example, the mould and die production as well as the aerospace industry. In addition, turbine blades and impellors are standard components machined by milling tools.

Gas combustion, nowadays, represents the world's second principal way of electricity generation. The conversion of natural gas in electricity is made through gas turbines, and the rotors of its compressors are components that present significant difficulty in its manufacture (Souza, 2006).

The high cost of manufacture led the gas turbine industry to look for high performance machining techniques for difficult-tocut materials like titanium and nickel alloys. Although considerable progress was obtained in the past regarding the machining rate, the results remain behind expectations. The poor cutting performance is mainly caused by the low thermal conductivity and extremely high thermal stability of these materials (Klocke et al., 2004).

Computerized Numerical Control (CNC) based five-axis milling machines offer new possibilities for optimizing the machining process. However, in contrast to the three-axis process, this practice is more complicated and generating the $\mathrm{NC}$ programs is still under development, particularly in case of machining complex free-formed surfaces. To program these NCpaths, standard CAM (Computer Aided Manufacturing) systems are usually applied. But these generated tool paths are often not satisfying, especially regarding the simultaneous movement of all tool axes (Zabel et al., 2006)

Paper accepted November, 2009. Technical Editor: Anselmo Eduardo Diniz
In the five-axis process, the three translational degrees of freedom of conventional three-axis milling are extended by the tilting and the rotational angle of the tool spindle or the machine table (Begau et al., 2008; Biermann et al., 2007; Weinert et al., 2001). This multi-axis concept results in several advantages: milling tools can be used in areas that are difficult to access, e.g. deep cavities, steep walls, or undercuts (Begau et al., 2008).

In order to utilize the potential of the five-axis milling process, this paper focuses on an optimization approach of the 5axis milling of titanium blisks, based on all the CAD/CAM modeling chain. In this development, special interest is pointed to the systematic analysis of the correlation between machining parameter and surface integrity properties.

\section{Nomenclature}

$$
\begin{aligned}
& \text { A = axial direction } \\
& \mathrm{a}_{\mathrm{p}} \quad \text { = axial depth-of-cut, } \mathrm{mm} \\
& \mathrm{a}_{\mathrm{e}} \quad=\text { radial depth-of-cut, } \mathrm{mm} \\
& \mathrm{C} 0=\text { surface parametric continuity, order zero } \\
& \mathrm{C} 1=\text { surface parametric continuity, order one } \\
& \mathrm{C} 2 \text { = surface parametric continuity, order two } \\
& \mathrm{G} 0=\text { geometric continuity, order zero } \\
& \mathrm{G} 1=\text { geometric continuity, order one } \\
& \mathrm{G} 2=\text { geometric continuity, order two } \\
& \mathrm{R} \quad=\text { radial direction } \\
& \mathrm{R}_{\mathrm{a}} \quad=\text { arithmetic average roughness, } \mu \mathrm{m} \\
& \mathrm{R}_{\mathrm{z}} \quad=\text { average maximum height of profile roughness, } \mu \mathrm{m} \\
& \mathrm{S} \quad=\text { surface } \\
& \mathrm{u} \quad=\text { direction in the parametric space } \\
& \mathrm{v} \quad=\text { direction in the parametric space } \\
& \mathrm{v}_{\mathrm{c}} \quad=\text { cutting speed, velocity, } \mathrm{m} / \mathrm{min}
\end{aligned}
$$




\section{$21 / 2$ or $3+2$ Axes Rough Milling}

Roughing involves removing stock from a user-defined blank plane by plane from top to bottom, thereby approaching the desired target geometry in a terraced representation. To ensure major uniformity of cutting forces on the tool teeth, rough milling is usually performed in $3+2$ axes. In case of 5 -axes machining centers 2 axes, the rotary ones, are used to fix distinct orientations between tool and workpiece that allows better conditions to the operation that is carried out by the other 3 axes, defining the term $3+2$ axes milling.

The major complexity factors of roughing stage are: reduced space between blades, in the case of solid rotors, what requires slender tools; and the materials generally used in these components. The most commonly used materials are austenitic stainless steel, titanium alloys and nickel based superalloys, all of them known as poor machinability materials due to properties like high toughness and ductility, low thermal conductivity and, in the case of titanium alloys high chemical affinity with tool materials in general. Theses variables aren't linear; moreover, during machining, their interactions make machining even harder.

This picture becomes even worse if materials proneness to chatter is accounted for. The maximum level of tolerable vibration in machining operations depends on their application. In rough operations this maximum tolerable vibration is mainly determined by its effects on tool life (Altintas, 2000).

To evaluate these factors the roughing of a TiAl6V4 titanium alloy impeller was taken as example and its blades were machined with different tool engagement conditions for a $4 \mathrm{~mm}$ diameter tool with four teeth and $22 \mathrm{~mm}$ of free length (Fig. 1).

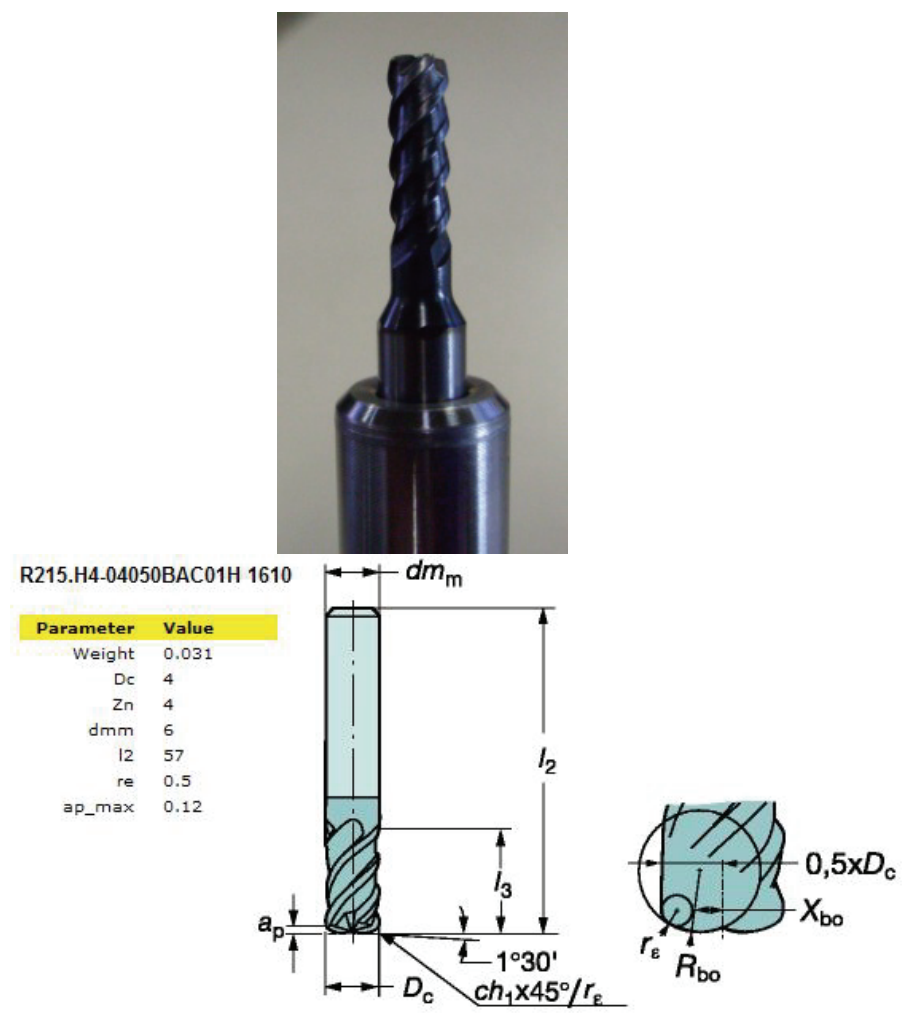

Figure 1. Cutting tool used in the roughing tests.

Cutting parameters assignment followed tool manufacturers' catalogs that recommended cutting speed $\left(\mathrm{v}_{\mathrm{c}}\right)$ of $50 \mathrm{~m} / \mathrm{min}$, axial depth-of-cut of $\left(a_{p}\right) 0.5 \mathrm{~mm}$ and feed per tooth $\left(f_{z}\right)$ of $0.1 \mathrm{~mm}$ and climb milling.

Chosen roughing strategy was trochoidal, with width of $70 \%$ of tool's diameter and step ahead equal to radial depth of cut. Chosen move standard was following the surface periphery (follow periphery) in $2 \frac{1}{2}$ axes prioritizing material removal on the work plane (level first) instead of on the depth (depth first).

The parameter that varied in these operations was the radial depth of cut $\left(a_{e}\right)$, and automatically the trochoidal step ahead, that assumed two values, $30 \%$ and $68 \%$ of tool's diameter, to configure two different engagement conditions, according to following arguments:

- For $30 \%$ radial depth of cut $\left(\mathrm{a}_{\mathrm{e}}\right)$, refrigeration conditions are well favorable. However, once the tool and impeller's geometric features have low stiffness, it is expected that the negative effects of vibrations supplant the lower thermal charge provided the restricting tool life (Antonialli, 2009);

- For $68 \%$ radial depth of cut $\left(\mathrm{a}_{\mathrm{e}}\right)$, engagement conditions are more favorable once mechanical requests are applied to the tool axis preserving its corner and increasing cutting stability. The negative factor would be the higher cutting forces added to higher thermal generation rate, due to chiptool contact length increase, besides more severe thermal chocks provoked by cutting fluid jets directed to the cutting edge;

- Two different typical roughing conditions were also considered, one with adjacent spaces between blades still with material (not machined), providing support to the blades being machined; and the other one with adjacent spaces between blades already machined by pervious operations (Fig. 2)

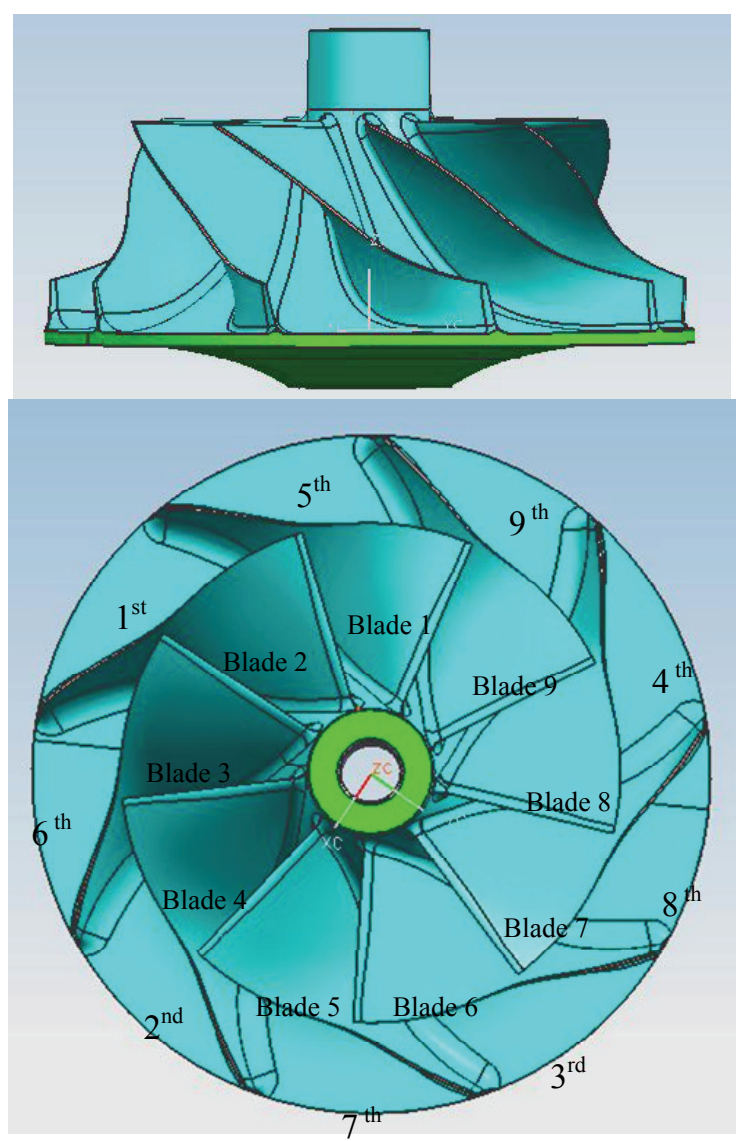

Figure 2. Roughing sequence of cavities between blades. 
Spindle power, sound pressure and tool flank wear were taken as indicators for this roughing evaluation. Spindle power data were acquired directly from the machine PLC through a Labview routine and the sound pressure was measured with a unidirectional $1 / 2$ inch microphone. The microphone was placed near the cutting zone and the audio signal was analyzed in time and frequency domain using the dynamic signal analyzer Data Physics Signal Calc ACE. Tool flank wear was evaluated with the aid of a stereoscopic magnifier Leica Wild M3C linked with a CCD camera, and the software Image Tool was used for capturing images and measuring the wear.

The tests showed that the presence of material providing support to the adjacent blades being machined exerts significant influence on consumed spindle power, as could be seen in Fig. 3. Nevertheless, spindle power consumption is less sensitive to radial engagement variations due to the small tool diameter that implies in small uncut chip sections.

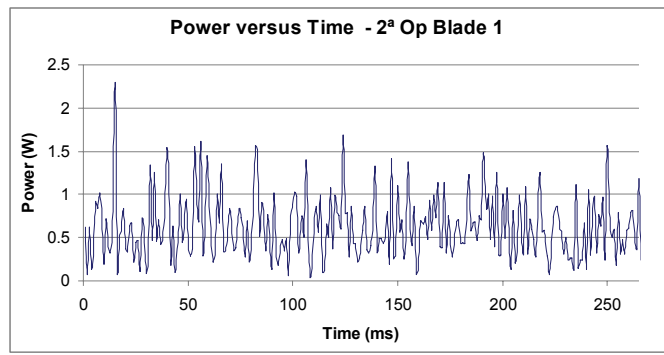

(with adjacent support) ae $=30 \%$

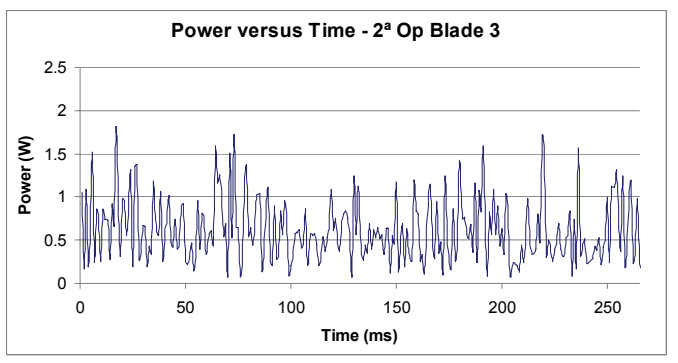

(with adjacent support) ae $=68 \%$

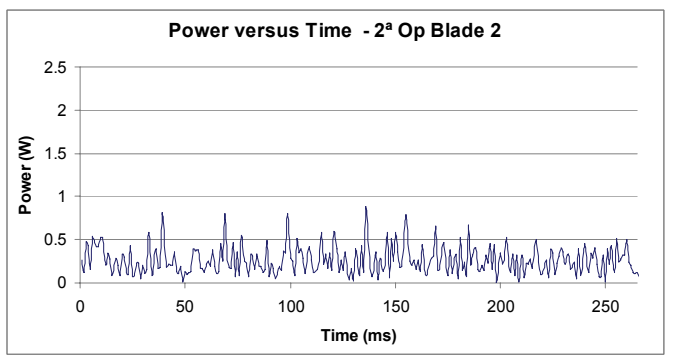

(without adjacent support) ae $=30 \%$

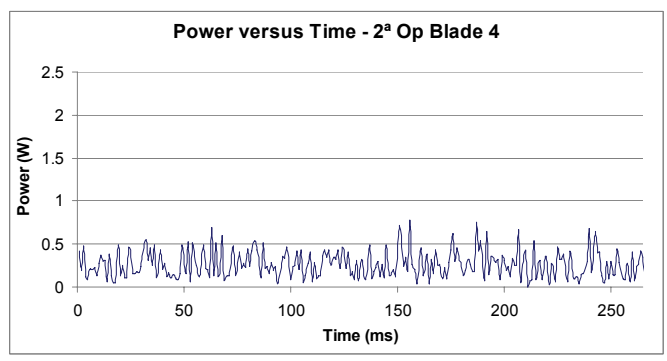

(without adjacent support) $\mathrm{ae}=68 \%$

Figure 3. Spindle power consumption for blades with and without adjacent support.
Owing to material's ductility and wall thickness resulted of adjacent cavities machining, high blade vibration occurred resulting in the removal of lower material amount and sometimes in cutting interruption. When there is material in adjacent cavities providing support and stability to the operation, material is more effectively removed, what rises consumed spindle power.

Considering milling operations lower power consumption not necessarily means better cutting performance. Not only the previous graph proves it, but also the sound pressure does so. Figure 4 shows that when there was no material in adjacent cavities, lesser consumption condition, more frequencies were excited, unlike the situation with support that rendered the biggest amplitude, $256 \mathrm{~Hz}$. However, the gradient of the loads is more important than their magnitude, what makes the first condition less stable than the latter. It was not noticed significant difference in the sound pressure between the distinct radial engagement conditions.

Hence, in the case of titanium impellers cavities roughing, the loss of material (and support) resulted of previous operations is a factor that causes more instability than engagement conditions imposed, determined by the cutting parameters. This can also be noticed by the tool wear behavior during roughing tests, exposed in Fig. 5.

Although the fact that larger radial depths of cut always lead to higher thermal charges due to: the bigger time that teeth spend engaged for every tool rotation; and the higher heat generation rate, once chip thickness raises with radial depth of cut $\left(a_{e}\right)$ increase; vibrations are more harmful to the tool, as can be noticed when the machining of blades with and without adjacent support are compared.

\section{5-Axes Finish Milling}

Geometries typically found in gas turbines rotor blades (as in impellers and BLISKs) impose important constrains to their modeling and manufacturing. Their arrangement in the disk periphery has a relative inclination with the plane that radially sections the disk. Besides, the blade could be torsioned around its axis resulting in concaves and convexes surfaces. Due to the great number of blades, only a little space with short angles remains between them, restricting accessibility and raising the necessity of slender end mills (Wülbeck, 2001). Take as example the simultaneous 5-axes milling of the bladed disk shown in Fig. 6.

In simultaneous 5-axes milling the cutting tool orientation calculation is made in relation to the surface normal vectors and these are the vector product of Eq. (1):

$$
\frac{\partial s}{\partial u} \times \frac{\partial s}{\partial v}
$$

In the $\mathrm{CAD} / \mathrm{CAM}$ process a solid object is frequently represented by a combination of two or more generic geometric entities. Thus, predefined parametric surfaces are usually subjected to Boolean operations of trimming and extending. This type of manipulation involves, in many times, intersection curves that do not coincide with the original isoparametric curves (Yang, 2003), what results in inadequate trajectories. In this case, a surface reparametrization is necessary.

For tool path generation and selection of CAM strategies for simultaneous 5-axes milling, the success of the operation has strong relation with the quality of CAD geometry generation. Both tasks require lots of practice of system users. The manufacturing modeling process consists of three main stages as follows. 


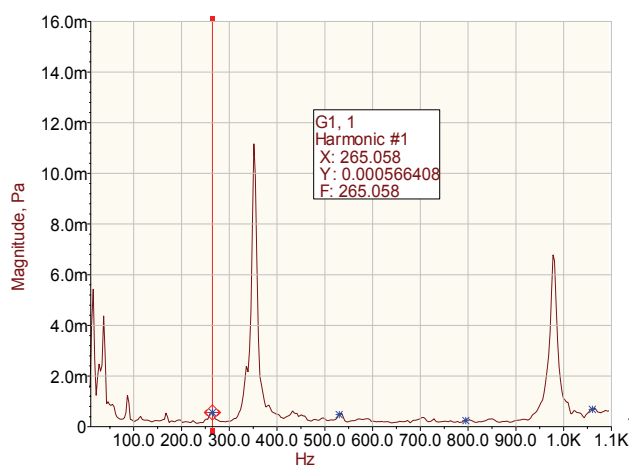

(With adjacent support) ae $=30 \%$

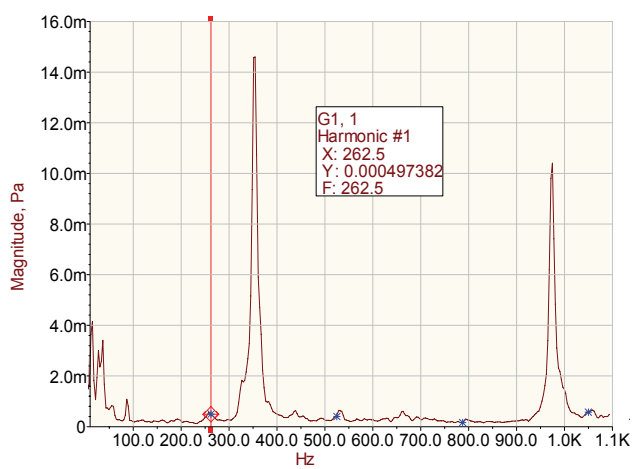

(With adjacent support) ae $=68 \%$

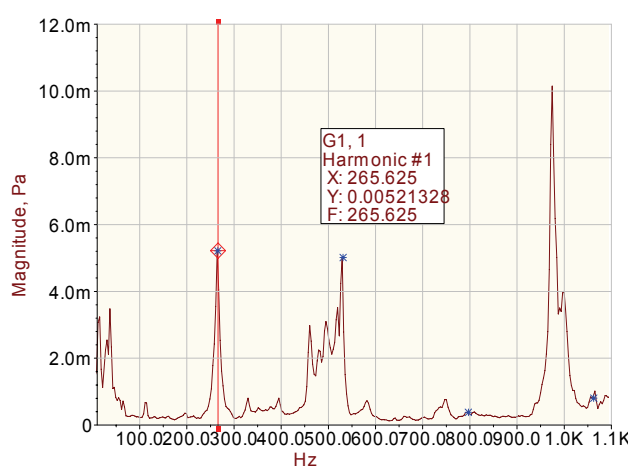

(Without adjacent support) $\mathrm{ae}=30 \%$

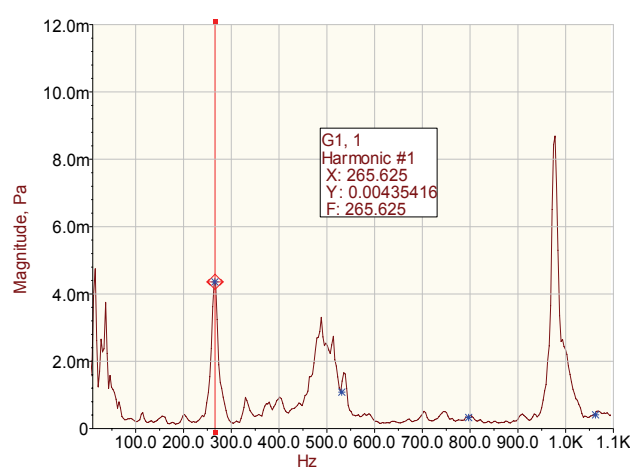

(Without adjacent support) $\mathrm{ae}=68 \%$

Figure 4. Sound pressure for blades with and without adjacent support.

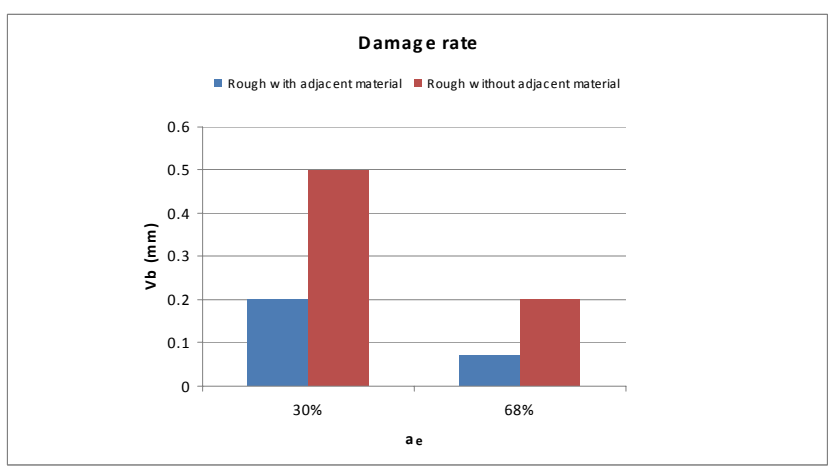

Figure 5. Tool wear evolution during roughing tests for blades with and without adjacent support.

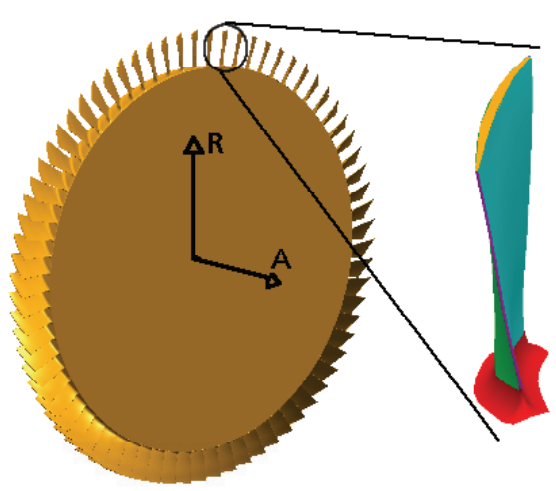

Figure 6. Bladed disk schematic representation with a blade in detail.

\section{Modeling for Simultaneous 5-axes Milling}

The first stage is the virtual modeling by CAD systems of the surfaces that will be 5-axes machined, where parametric and geometric continuity is elementary.

Parametric continuity is defined by the coherence between adjacent surface patches. Patches are surface segments defined by two sets of four splines arranged like rows and columns. These curves are usually of $3^{\text {rd }}$ degree for open surfaces and $5^{\text {th }}$ degree for closed surfaces like corners. Complex parametric surfaces are sets of patches in an amount proportional to their complexity. The more complex the surface, the bigger the number of patches that builds it. And with higher amount of patches concordance among them becomes more difficult, what in turn makes surfaces less smooth rendering wavy machined surfaces. Hence, rationalizing the number of patches of a surface constitutes a requirement for surface modeling.

Surface parametric continuity can be of three different orders, $\mathrm{C} 0, \mathrm{C} 1$ and $\mathrm{C} 2$, according to the kind of transition between patches. $\mathrm{C} 0$ transition is marked by single coincidence of boundary curve - two adjacent patches with the same curve as boundary - defined by $\mathrm{C}(v)$, and along this curve for every point there are two vectors $\delta \mathrm{S} 1 / \delta \mathrm{u}$ and $\delta \mathrm{S} 2 / \delta \mathrm{u}$ with different directions each one tangent to one patch. In $\mathrm{C} 1$ transition order not only the boundary curve is coincident, but also the first derivates of the patches are, this means that vectors $\delta \mathrm{S} 1 / \delta \mathrm{u}$ and $\delta \mathrm{S} 2 / \delta \mathrm{u}$ have the same direction. What distinguishes $\mathrm{C} 1$ and $\mathrm{C} 2$ transition orders is that for the latter the magnitude of cited vectors is equal.

Geometric continuity regards surfaces transitions. For it there are also three orders, G0, G1 and G2, with similar definitions to parametric continuity orders, but applied to surfaces. 
When modeling surfaces for simultaneous 5-axes milling attention should be paid to the smoothness of the transitions, which has to be at least of $\mathrm{C} 1$ and G1 order and preferably of $\mathrm{C} 2$ and G2. Once in 5-axes milling tool axis is oriented by surface normal vectors, continuity of $\mathrm{C} 0$ or $\mathrm{G} 0$ order imply in two distinct normal vectors for the same point, disorientating the tool and causing abrupt decelerations and tool axis orientation changes, configuring what is known as jerks or jerky movements. When machining HRSA and other materials of poor machinability, this can drastically diminish tool life, beside the fact that it undoubtedly causes gauge marks that can condemn the machined piece.

Hence, patches and surfaces should be necessarily aligned. This alignment defines connection points for parametric curves thus controlling surface form in a certain extension. Parametric alignment should be chosen in a way that does not distort the surface, then avoiding curvatures in erratic directions and manufacturing problems.

Take a flank milling operation as example. This operation, usually applied to rotors machining, can be really problematic when there is no care with patches and surfaces continuity. What generally happens is that the contact of mill's periphery and piece surface cannot be guaranteed during the whole tool path.

In the modeling process a solid body is frequently represented by a combination of two or more primary geometric entities. In this way, predefined parametric surfaces usually are subjected to Booleans operations of trimming and extending. Generally, intersection curves resulted of these Boolean operations do not match the original isoparametric curves. The result is that the remaining original isoparametric curves are not adapted to the boundary anymore. Consequently, in tool path generation process these surfaces could generate unsatisfactory tool paths (Yang, 2003).

Therefore, the task of trimmed surfaces reparametrization is fundamental if this geometry is used in a CAD/CAM chain. The algebraic interpolation method or bi-linear method (Coons method) (Farin, 1993; Rogers, 1990) and the Laplace method (Yang, 2003; Lehtimäki, 1999) are the two principal methods, the first due to its simplicity and the latter because it solves some problems of the first. In both methods the 2D parametric dominium of the trimmed surface is reparametrized in a new 2D parametric dominium. After this tool paths are generated following the new parametrization set.
Figure 7 shows a tool path generated using an IGES model that was reparametrized by Coons method. The figure shows emphasized regions where tool path errors occurred due to reparametrization algorithm inefficiency and mismatch of isoparametric curves in the trimmed surface boundary.

\section{Tool Path Generation}

The second stage consists in generating the tool path in accordance with the commercial CAM strategy chosen. In this stage are chosen cutting tools, tool-machine, type of milling (end or flank milling), direction of cut, machining parameters and interpolation tolerances. After this, algorithms calculate a set of locations and trajectories for the tool (Fig. 8).

The calculation of simultaneous 5-axes milling tool paths can be dismembered in two tasks, CC (Cutter Contact) and CL (Cutter Location) definition, and tool axis orientation in relation to machined surfaces.

First, points of contact of the tool in the surface to be machined (CC) and their sequence are defined and their posterior conversion to cutter location points $(\mathrm{CL})$ is made, what corresponds to the path distribution along the surface and definition of the feed direction. The most common way of doing that for 5-axes milling is applying an isoparametric method. For the isoparametric method the tool path generation depends on $u$ and $\mathrm{v}$ parameters of the parametric surface. The main advantage of this method is its low mathematic complexity. However, as the intervals between adjacent steps are constant in the parametric space, in the three-dimensional space this can diminish or increase, what leads frequently to the generation of redundant trajectories. Another inconvenience of this method is its high dependency on the mode of surface construction and its discretization grade (Gomes, 2005).

After CC and CL determination, tool axis orientation is defined, and the method applied here by commercially available CAM systems is the Sturz Method, also known as inclined tool method. This consists of orienting tool axis with a constant inclination in relation to surface's local normal vector. Two angles have to be defined, sturz angle $\beta$, in the feed direction, and attack angle $\alpha$, orthogonal to that direction, as shown in Fig. 9. The smaller these angles are, the bigger is the risk of gouging, but, on the other hand, increasing these results in bigger cusps and consequently worst surface quality, making additional passes necessary (Souza, 2006).

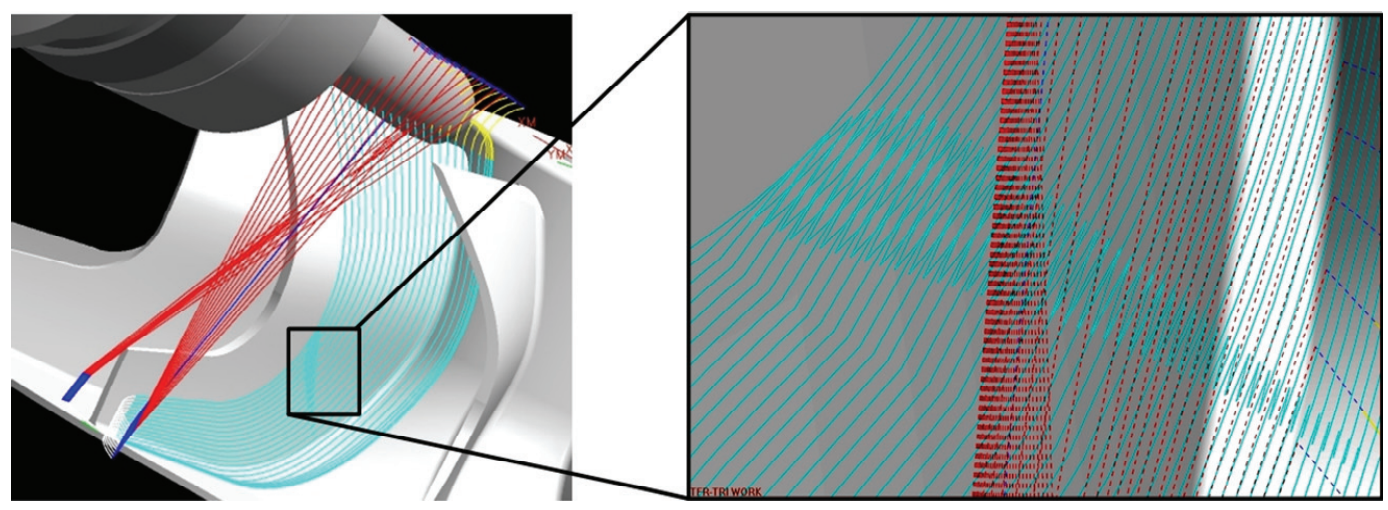

Figure 7. Errors in a tool path generated using an IGES model that was reparametrized by Coons method. 


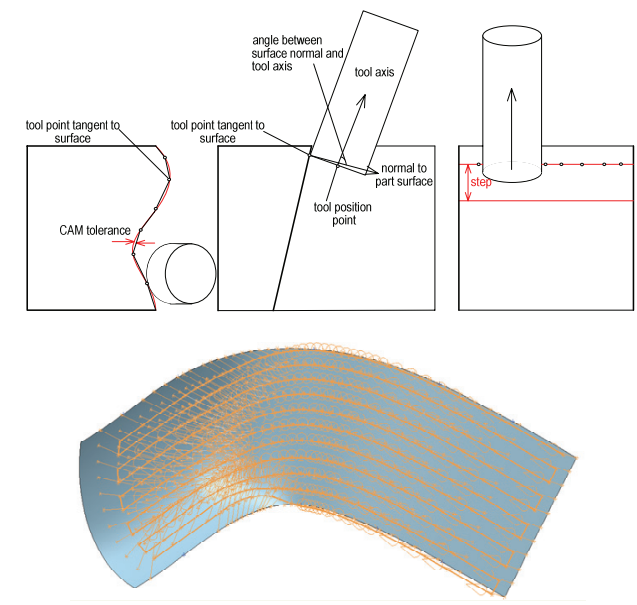

Figure 8. Considered parameters for simultaneous 5-axes milling tool path generation.

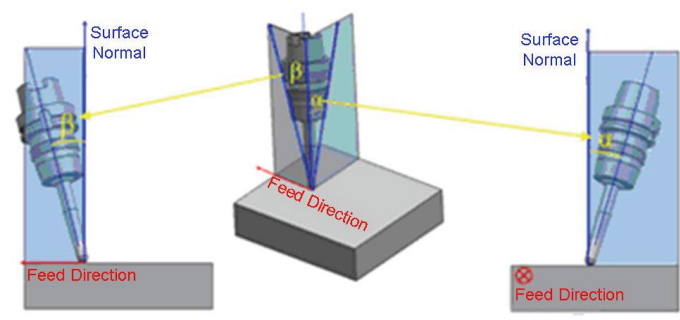

Figure 9. Programmable inclination angles in the Sturz Method.

Although it has proved higher efficiency over 3-axes milling for freeform surfaces machining, this is a trial and error method, where several angles are tested until the littlest possible inclination with no gouge is achieved. It makes this method very user dependent and subordinated to the graphics simulation system reliability, once gouge and collisions can only be identified through these systems. Minute checks of generated tool paths are needed and, if gouges are identified, the path must be regenerated. These steps make tool path generation a time consuming stage and generated path usually needs optimization.

\section{Program Execution in a Machine Tool}

The last stage is to perform the program in a machine tool. The example now is the simultaneous 5-axes milling of a TiAl6V4 five blades section of a BLISK (Fig. 10). There was no need for the complete piece, because this five blades section contains the main manufacturing constrains with its geometrical complexity, limited accessibility and poor machinability.

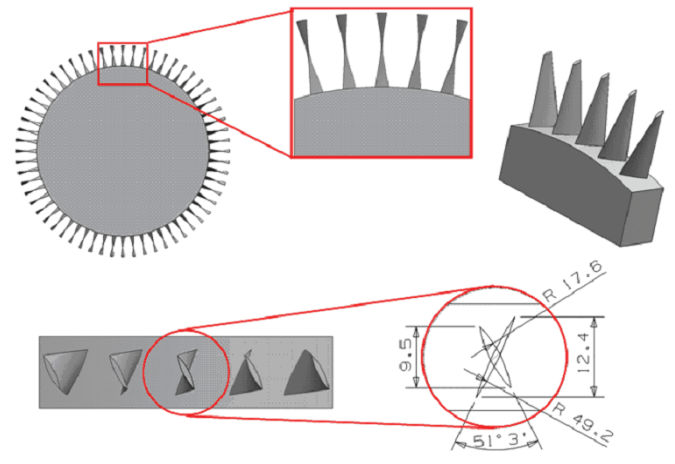

Figure 10. Five blades test-piece.
Figure 11 shows the set-up of the blisk section and its fixture. This configuration was conceived in order to give more realness to the operation making the rotary moves of the machine as close as possible to that found in a real blisk machining.

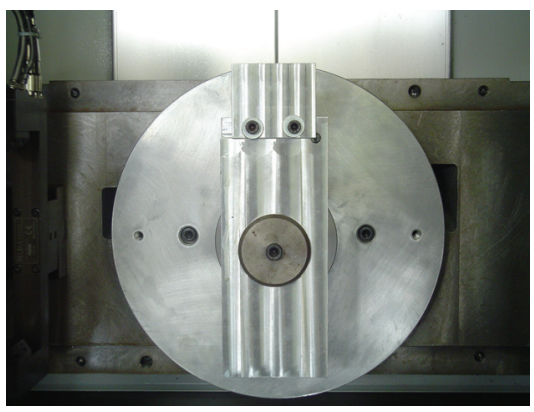

Figure 11. Blisk section fixturing se-up configuration.

A $12 \mathrm{~mm}$ diameter solid carbide ball-nose mill with TiAlN PVD coating, shown in Fig. 12, was used to finish the blades.

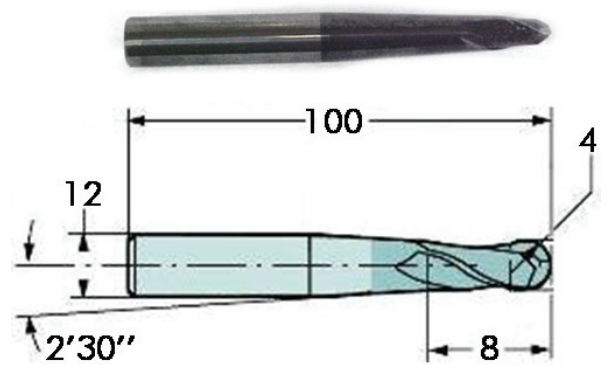

Figure 12. Solid carbide ball-nose mill used for test-piece finishing.

The main objective of this analysis was to find the strategy that best fits for the machining of a turbine BLISK blade. Thus, once the focus was the finishing operation, roughing and semi-finishing operations were performed according to finishing strategies applied. Considering that the blade is sectioned in equivalent portions along the foot-to-head direction, the following strategy hypotheses were raised:

a) total roughing and semi-finishing of a blade side followed by its finishing and, after that, total roughing, semi-finishing and finishing of the opposite side;

b) direct finishing, without roughing and semi-finishing, divided into four stages with both sides of the blade being machined alternately;

c) machining of the blade divided into two stages. First both sides of the blade are roughed and semi-finished before their finishing. In the second stage the same sequence is repeated for the other half of the blade;

d) similar to c, but with the blade divided into three sections machined in three stages;

e) total roughing and semi-finishing of both blade sides for only then finishing it with the mill contouring it.

Comparison between the strategies was made mainly confronting surface roughness and machining time. But process rigidity was taken into account through observation of possible chatter marks and burrs.

Roughing operations of the space between blades were made with $3+2$ axes milling, looking forward process rigidity and security. Semi-finishing operations used the same strategies adopted for finishing operations, only modifying the tool and increasing step 
increment and CAM tolerance. The stock left for the finishing operation was $0.5 \mathrm{~mm}$ for all blades.

For tool path generation of all blades finishing the method for define CCs and CLs was isoparametric and for the orientation the Sturz Method was used. The parametric curves chosen to determine the tool movement pattern were the ones in the $u$ direction of the surface shown in Fig. 13, because these curves permitted the best accessibility.

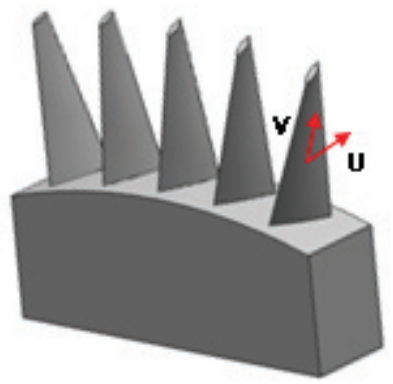

Figure 13. Direction of $u$ and $v$ curves of the blades surfaces parametric field.

The same CAM strategy was used for the first to fourth blades. The discrepancies were the number of stages used, machining operations order and, for the second blade, the change of the surface speed due to the fact that roughing and semi-finishing was not used. The pattern of the tool paths generated can be checked in Fig. 14.

To allow complete and uniform machining of the blades, it was necessary to create auxiliary surfaces for each side of it and to extend them in the $u$ and $v$ directions. These auxiliary surfaces were used as drive surfaces for the tool path generation.
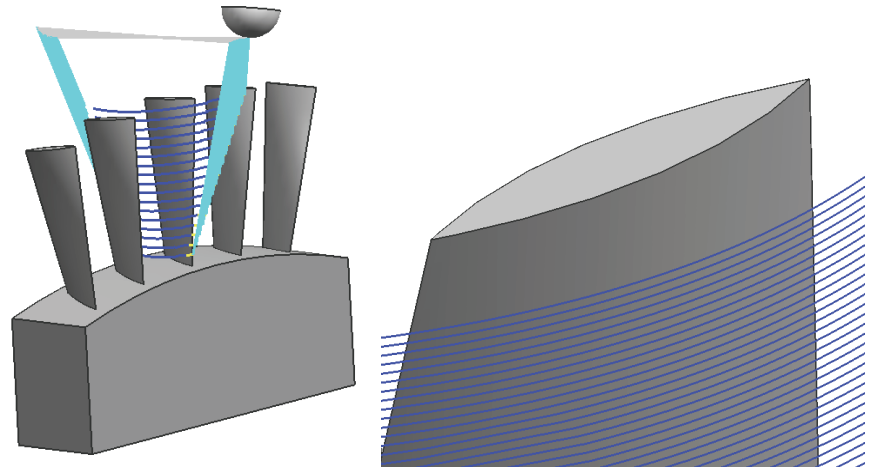

Figure 14. Tool path pattern for the first to fourth blades.

In all tool paths the step increment adopted was $0.2 \mathrm{~mm}$ and $\beta$ and $\alpha$ angles were $0^{\circ}$ e $70^{\circ}$ respectively, the latter being defined through graphic verifications by trial and error method until the littlest possible inclination with no collision with the next blade was achieved. Surface speed was $65 \mathrm{~m} / \mathrm{min}$ for second blade and 120 $\mathrm{m} / \mathrm{min}$ for the others, while $0.1 \mathrm{~mm}$ feed per tooth and climb milling were adopted for all cases. A $30 \%$ feed rate decrease was programmed for entrance and exit moves.

Finishing of all blades feet was made in the same way, the only feasible way. The method was floor surface machining, which in this case is the cylindrical surface of the disk where blades are fixed. The tool flank is guided by the wall surfaces, the blade surfaces for this case, as indicated by Fig. 15. In this figure there is an image of the graphic verification of this operation two neighbor blades.

The tool paths for the first and fifth blades were verified, simulated (Fig. 16) and validated in intermediate blades before being machined in the test-piece.

After machining operations roughness measures were taken in six points of each blade, one in the top, one in the middle and one in the bottom, on both blade sides, as shown in Fig. 17. In this figure it also can be noticed how the test-piece was fixed to an indexing head to allow roughness measures.

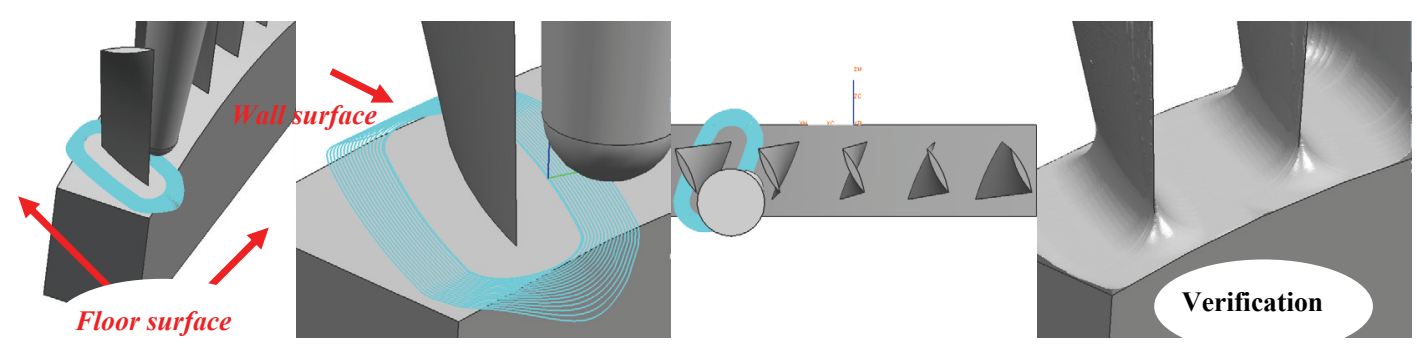

Figure 15. Blades' foot finishing tool path.
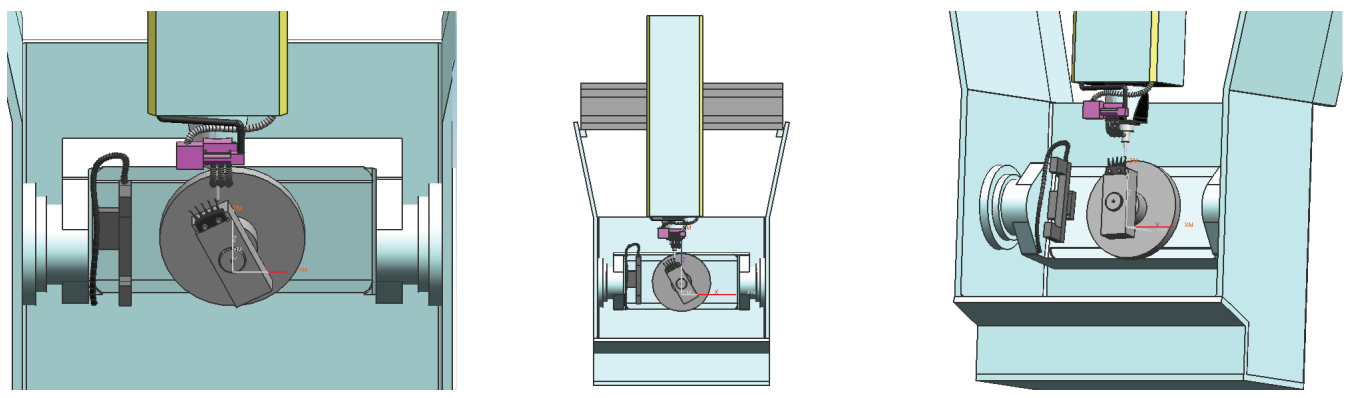

Figure 16. Simulation of machining center axes motions for the test-piece machining. 


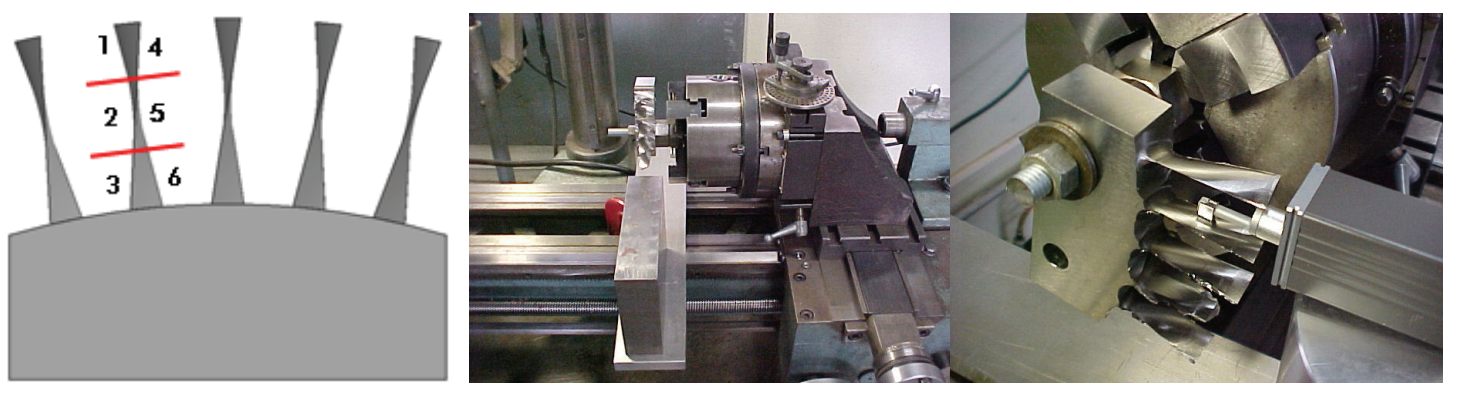

Figure 17. Roughness measure points and test-piece set-up for the measures.

Table 1. BLISK blades $\mathrm{Ra}$ and $\mathrm{Rz}$ roughness.

\begin{tabular}{|r|c|c|c|c|c|c|c|c|c|c|}
\cline { 2 - 12 } \multicolumn{1}{c|}{} & \multicolumn{2}{c|}{ Blade 1 } & \multicolumn{2}{c|}{ Blade 2 } & \multicolumn{2}{c|}{ Blade 3 } & \multicolumn{2}{c|}{ Blade 4 } & \multicolumn{2}{c|}{ Blade 5 } \\
\cline { 2 - 12 } \multicolumn{1}{c|}{} & Ra & Rz & Ra & Rz & Ra & Rz & Ra & Rz & Ra & Rz \\
\hline 1 & 0.50 & 2.66 & 0.65 & 2.81 & 0.64 & 4.00 & 0.72 & 4.32 & 0.66 & 3.83 \\
\hline $\mathbf{2}$ & 0.46 & 2.44 & 0.54 & 2.55 & 0.58 & 3.65 & 0.74 & 3.81 & 0.45 & 2.72 \\
\hline 3 & 0.74 & 4.00 & 0.80 & 4.26 & 0.71 & 3.79 & 0.96 & 4.85 & 0.80 & 4.39 \\
\hline Left & 0.57 & 3.03 & 0.66 & 3.21 & 0.64 & 3.81 & 0.81 & 4.33 & 0.64 & 3.65 \\
\hline 1 & 0.74 & 3.69 & 0.50 & 2.53 & 0.74 & 4.22 & 0.87 & 4.89 & 0.69 & 3.90 \\
\hline 2 & 0.50 & 3.11 & 0.46 & 2.37 & 0.95 & 5.30 & 0.60 & 3.56 & 0.76 & 3.99 \\
\hline 3 & 1.04 & 6.05 & 0.80 & 4.12 & 0.80 & 4.64 & 1.39 & 6.62 & 0.66 & 3.47 \\
\hline Right & 0.76 & 4.28 & 0.59 & 3.01 & 0.83 & 4.72 & 0.95 & 5.02 & 0.70 & 3.79 \\
\hline$\sigma$ & 0.223 & 1.313 & 0.150 & 0.852 & 0.130 & 0.614 & 0.279 & 1.093 & 0.122 & 0.571 \\
\hline Mean & 0.66 & 3.66 & 0.63 & 3.11 & 0.74 & 4.27 & 0.88 & 4.68 & 0.67 & 3.72 \\
\hline Values in $\mu \mathrm{m}$
\end{tabular}

Measured $R_{a}$ and $R_{z}$ roughness are disposed in Tab. 1 and mean values of $R_{a}$ for each blade are also presented in the form of bars graph in Fig. 18, in which confidence intervals are associated with the means for better comparison between the results.

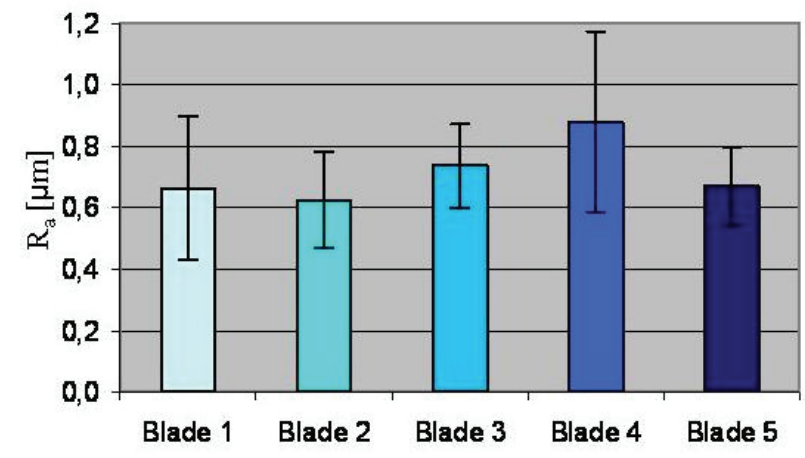

Figure 18. BLISK blades Ra roughness.

The surface quality achieved was satisfactory in a general point of view, never surpassing $1 \mu \mathrm{m} \mathrm{R}_{\mathrm{a}}$. Comparing blades roughness among them it is only possible to tell that the roughness of the second blade was lower than of the fourth blade.

First blade, for which all three machining stages, roughing, semi-finishing and finishing, were performed in one of the sides of the blade before starting to machine the other side, rendered good surface finishing, principally on the first machined side, when there still was material in the adjacent space between blades. Nevertheless, there were gouging marks over the surface of which probable origin was the tool paths generated by the CAM system (Fig. 19).

When machining the opposite side of this blade noise could be heard, due to the lack of rigidity of the slender geometry being machined, chatter marks were present in the top of the blade and burrs were also left.
To avoid these problems titanium thin walls (as turbine blades are) must be milled with alternated blade side passes with step increment of 0.5 to 2 times the mill diameter (Sandvik, 2004). Strategy used for the third and fourth blades followed this advice. Machining was divided into two stages for the third blade and for the fourth blade there were used three stages, expecting higher rigidity once the length of the slender feature is reduced.

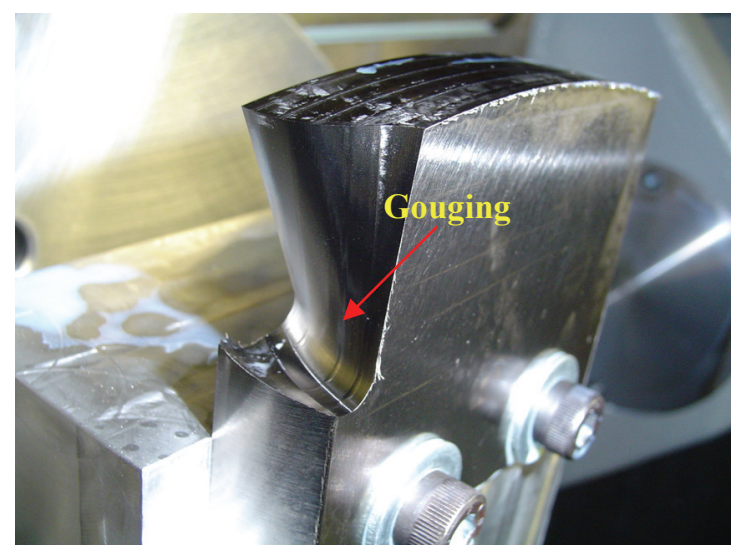

Figure 19. Left side of first blade after finishing.

However, chatter marks in the blade's top and burrs appeared in these blades (Fig. 20). Dividing the blades only diminished the noise level and burr amount, but for the roughness it cannot be said that there was improvement. For the second blade, of which roughness had the lowest values, the strategy did not consider advices of applying at least a roughing stage before the finishing operation (Aronson, 2005; Zelinsky, 2005). Due to the lack of accessibility, and to privilege rigidity, this operation was divided into four stages with the blades sides being machined alternately. 
As expected, eliminating the roughing stage made the operation really severe and with the presence of chatter, what made the piece fixturing slightly rotate.

The fifth and last blade had its two adjacent spaces roughed and semi-finished before the finishing operation. Then, the tool milled the blade contouring it, hence following the advices of pre-machine the piece and of milling titanium thin walls with alternated blade side passes with step increment of 0.5 to 2 times the mill diameter.
Nevertheless, this strategy was not able to eliminate chatter marks in the top of the blade, but rendered good surface finishing and minimum amount of burr.

Evaluation of the machining of the blades' feet can be made with the picture of Fig. 21 which shows the foot of the fifth blade. Once the strategy was the same for all blades, the good surface quality was similar for the other blades.

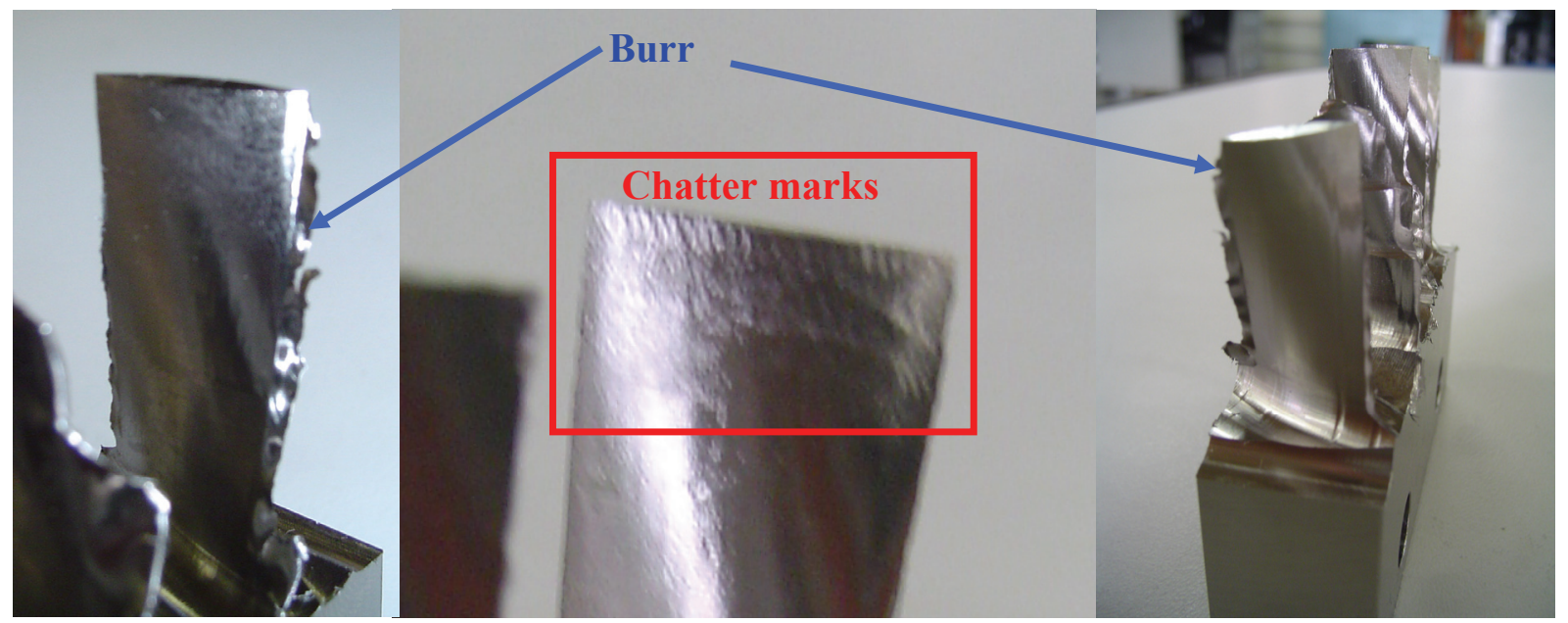

Figure 20. Burr and chatter marks after milling.

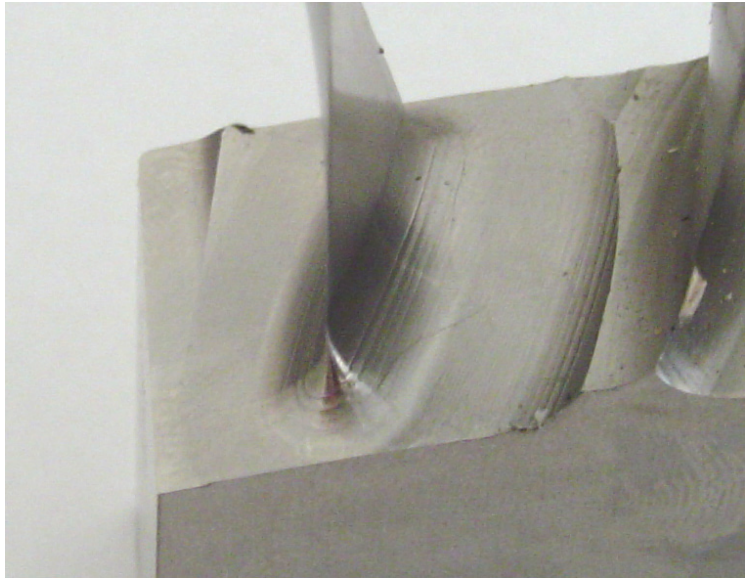

Figure 21. Fifth blade foot after its finishing.

Regarding machining time, fifth blade presented the best result only accounting for finishing operation with machining time of about 10 minutes. This is followed by the first, third and fourth blades with about 23 minutes while the second blade took about 42 minutes due to the lower surface speed applied.

However, if considering all secondary times like tool changes, rapid movements, adjusting movement and the time took by roughing and semi-finishing, total time for the second becomes lower than for the fifth blade, however, it is supposed, in detriment of tool life. Thus, a definitive conclusion about if it is advantageous to leave roughness and semi-finish behind only would be possible if associated with a cost analysis considering tool life reductions.

Hence fifth blade strategy was the one that satisfied the most the involved requirements, with low roughness, relatively stable machining and relatively low machining time.

Finally, using software transforming tools and tool path copy, available in CAM systems, it is possible to apply this strategy for the machining of all blades of a complete rotor.

\section{Conclusion}

The goal of this work was to analyze constrains and requirements for the manufacturing of freeform surfaces usually applied to gas turbines components made of TiAl6V4 titanium alloy. The following considerations were raised:

- When roughing titanium alloy tool life is more influenced by higher contact times, and consequent higher thermal generation in chip-tool interface, not depending of first contact condition of the cutting edge.

- In impellers cavities roughing, due to small diameter tools and to characteristics of previously machined region surfaces, it is more significant to control chatter excited by adjacent geometries. These characteristics are even more significant than proper radial engage conditions of the cutting tool.

- In surface modeling for simultaneous 5-axes milling is elementary to concern with smooth direction transitions and uniformity of normal vectors of transition regions, as much in the surfaces (among patches) as among the surfaces.

- For geometries similar to that used in the finishing section of this work, the best option is to perform roughing and semifinishing operations of all spaces between blades before finishing them, with the tool contouring them and following parametric curves that are orthogonal or almost orthogonal to foot-to-head direction, $u$ curves in this case, and using Sturz Method with null Sturz angle and the smallest attack angle that permits space between blades.

\section{References}

Altintas, Y., 2000, "Manufacturing Automation", Ed. Cambridge University Press, New York, USA, 288 p.

Antonialli, A.Í.S., 2009, "Uma contribuição ao fresamento frontal da liga de Titânio Ti-6Al-4V", Doctoral Thesis, Universidade Estadual de Campinas, Campinas, Brazil, 116 p. 
Aronson, R.B., 2002, "What's different about manufacturing for aerospace? - quality and reliability are more important", Manufacturing Engineering Magazine, Vol. 128, in http://www.sme.org/cgi-bin/get-mag. pl?\&\&02mam050\&000007\&2002/02mam050\&ARTME\&SME\& (accessed on October 2005).

Begau, C. et al., 2008, in: Yang, A., Shan Y., Bui, L., "Success in Evolutionary Computation - Studies in Computational Intelligence" Ed. Springer, London, UK, $372 \mathrm{p}$.

Biermann, D. et al., 2007, "Intelligent process planning methods for the manufacturing of molds", Proceedings of PDMS International Conference on Product Design and Manufacturing Systems.

Farin, G., 1993, "Curves and Surfaces for Computer Aided Geometric Design”, Ed. Academic Press, New York, USA.

Gomes, J.O., Souza, G.O., Silva, A.S.A., 2005, "Simultaneous 5-axes and 3-axes milling comparison applied an a automotive component", Proceedings of COBEM International Congress of mechanical Engineering, CD-ROM, Ouro Preto, Brazil.

Klocke, F., Markworth, L., Messner, G., 2004, "Modeling of TiAl6V4 Machining Operations", Fraunhofer Institute of Production Technology, Aachen, Germany.

Lehtimäki, R., 1999, "Elliptic grid generation”, American Institute of Aeronautics and Astronautics Journal, Vol. 37, No. 6, pp. 768-770.
Rogers, D.F., Adams, J.A., 1990, “Mathematical Elements for Computer Graphics", Ed. McGraw-Hill, Singapore, 661p.

Sandvik Coromant, 2004, "Titanium Machining - Application Guide", AB Sandvik Coromant, Sandviken, Sweden.

Souza, G.O., 2006, "Fresamento em 5-eixos simultâneos de pás de compressores de turbinas a gás em TiAl6V4", Master's Dissertation, Universidade Federal de Santa Catarina, Florianópolis, Brazil, 168 p.

Weinert, K., Zabel, A., 2001, "Modeling, simulation and visualization of simultaneous five-axis milling with a hexapod machine tool", Proceedings of ESS - 13th European Simulation Symposium, Marseille, France, pp. 344-348.

Wülbeck, C., 2001, "Modellierung und bewertung von simultanmehrachsigen NC-bearbeitungsstrategien für komplexe integralbauteile der luftfahrtindustrie", Studienarbeit, Rheinisch-Westfälischen Hochschule, Aachen, Germany.

Yang, D.C.H., Chuan, J.J., Oulee, T.H., 2003, "Boundary-conformed tool path generation for trimmed free form surfaces", Computer-Aided Design, Vol. 35, pp. 127-139.

Zabel, A. et al., 2006, "Improvement of machine tool movement for simultaneous five-axes milling", Proceedings of CIRP, pp. 159-164.

Zelinsky, P., 2005, "Getting the time out of titanium", Modern Machine Shop, in http://www.mmsonline.com/articles/080103.html (accessed on October 2005). 